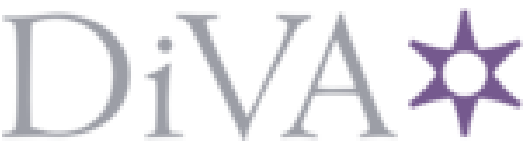

http://www.diva-portal.org

Postprint

This is the accepted version of a paper presented at Chinese Automation Congress (CAC), Jinan.

Citation for the original published paper:

Sun, B., Liyao, M., Wei, C., Wei, W., Prashant, G. et al. (2017)

An Improved k-Nearest Neighbours Method for Traffic Time Series Imputation

In: IEEE

N.B. When citing this work, cite the original published paper.

Permanent link to this version:

http://urn.kb.se/resolve?urn=urn:nbn:se:bth-15209 


\section{An Improved k-Nearest Neighbours Method for Traffic Time Series Imputation}

\author{
$1^{\text {st }}$ Bin Sun \\ Blekinge Institute of Technology \\ Karlskrona 37179, Sweden \\ bin.sun@bth.se
}

$4^{\text {th }}$ Wei Wen

Blekinge Institute of Technology

Karlskrona 37179, Sweden

wei.wen@bth.se

\author{
$2^{\text {nd }}$ Liyao Ma \\ School of Electrical Engineering \\ University of Jinan \\ Jinan 250022, China \\ cse_maly@ujn.edu.cn
}

\author{
$5^{\text {th }}$ Prashant Goswami \\ Blekinge Institute of Technology \\ Karlskrona 37179, Sweden \\ prashant.goswami@bth.se
}

\author{
$3^{\text {rd }}$ Wei Cheng \\ Kunming University of Science and Technology \\ Kunming 650093, China \\ Blekinge Institute of Technology \\ Karlskrona 37179, Sweden \\ Corresponding Author: wei.cheng@bth.se
}

\author{
$6^{\text {th }}$ Guohua Bai \\ Blekinge Institute of Technology \\ Karlskrona 37179, Sweden \\ guohua.bai@bth.se
}

\begin{abstract}
Intelligent transportation systems (ITS) are becoming more and more effective, benefiting from big data. Despite this, missing data is a problem that prevents many prediction algorithms in ITS from working effectively. Much work has been done to impute those missing data. Among different imputation methods, k-nearest neighbours (kNN) has shown excellent accuracy and efficiency. However, the general $\mathrm{kNN}$ is designed for matrix instead of time series so it lacks the usage of time series characteristics such as windows and weights that are gap-sensitive. This work introduces gap-sensitive windowed kNN (GSW-kNN) imputation for time series. The results show that GSW-kNN is $34 \%$ more accurate than benchmarking methods, and it is still robust even if the missing ratio increases to $90 \%$.

Index Terms-Traffic Time Series, Gap-Sensitive Windowed k-Nearest Neighbours (GSW-kNN), Missing Data Imputation
\end{abstract}

\section{INTRODUCTION}

Nowadays, intelligent transportation systems (ITS) are becoming more and more effective, benefiting from big data generated by modern sensors and devices [1]. Many prediction algorithms rely on such big data, for example, frequently used prediction methods, including auto regressive integrated moving average (ARIMA) [2], neural network [3]-[5], support vector regression (SVR) [6], kNN regression [7] among others. These methods cannot make full use of dataset in case of missing values [8]. While the amount of data captured by various devices is getting larger, the amount of missing data increases as well. The missing ratio of traffic data is usually $5 \%$ to $25 \%$, sometimes more than $90 \%$ [8]-[10].

There has been much work on imputation for time series data and kNN has produced imputations accurately and efficiently [8], [11]. However, two key topics are not found in the literature. Firstly, missing values lead to gaps within time series which should be considered during neighbour distance measurement. Secondly, time series is continuous on time scale which is not considered in general $\mathrm{kNN}$ imputation. To solve the problems mentioned above, this work proposes a gap-sensitive windowed kNN (GSW-kNN) to impute missing values in traffic time series.

\section{BACKGROUND AND RELATED WORK}

Data missing situation can be categorized into three types [12]-[14]. In the first type, the values are missing completely at random (MCR). The missing positions are completely unrelated with each other and are randomly located on the time scale. In the second type, the values are missing at random (MR) which means the missing positions are dependent on neighbouring samples. They are kind of clustered or continuous, but the clusters are randomly located on the time scale. The situation is usually due to device damage or maintenance. The third type is missing values not at random (MNR). The missing clusters are not randomly located on the time scale. There can be more types when considering spatial information such as multi-sensor station situation, which is out of the scope of this work [9].

To impute missing values, data imputation methods are developed which can be divided into three categories: prediction based, interpolation based and statistical/machine learning based [8], [15]. Prediction based methods, such as ARIMA and neural network, have the problem that they only make use of continuous chunks in the time series [8]. A typical interpolation based method is regression spline, which is a piecewise polynomial function that tries to approximate the unknown function [16], [17]. In the statistical/machine learning category, k-Nearest neighbours (kNN) [9], [18] and principal component analysis (PCA) based methods [8], [19] have shown great performance [8], [14] and efficiency [11]. It has been shown that different PCA based methods perform similar with regards to accuracy [14]. Another work introduces an ad hoc category which includes mean, median and last observation carried forward (LOF) [13]. Among those methods, $\mathrm{kNN}$ is accurate and efficient [8], [11]. 


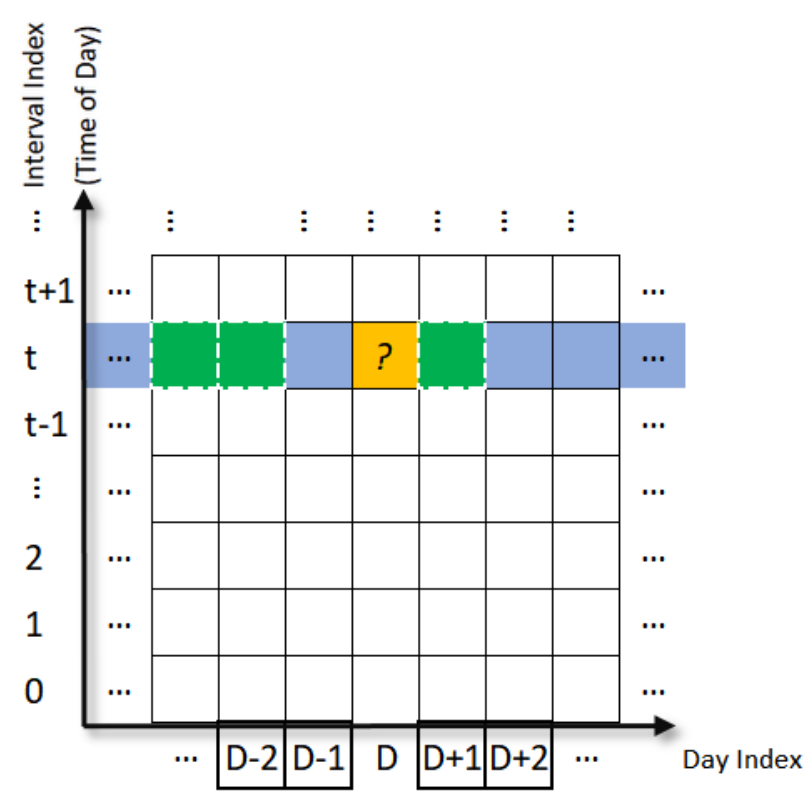

Fig. 1. General kNN imputation matrix. Imputation for time $t$ on day $D$ using other days' data. The entire data of each day as a vector is taken to calculate similarity and the most similar days are selected. Three dashed dark green squares are the selected nearest neighbours' data at $t$ of corresponding days $(k=3)$. 4 searchable history days (no shifts) lead to 4 potential neighbours.

The general kNN imputation of traffic data is shown in Fig. 1. One value at time $t$ on day $D$ is missing. Each searchable day in the history data is considered as a neighbour. The distance between the day $D$ and other days are calculated and the nearest $k$ days are selected as nearest neighbours. The values (white-dashed dark green squares) at time $t$ of the $k$ days are averaged to impute the missing value at time $t$ on day $D$.

One problem is the general kNN imputation is that it is developed for matrix such as microarrays in human genes, which is not considering traffic data on the time scale. An important characteristic of time series is correlation and continuous change on the time scale. A possible approach to make use of such a characteristic is to use window based shifting, which have been used for kNN based time series prediction [20], [21]. Though this approach works well, it has not been used for imputation.

Another problem is that we can see that the general kNN is not considering the gap of missing data. Gaps occur if any values are missing. When the general $\mathrm{kNN}$ algorithm tries to impute a value, it measures its distance to neighbours with the positions of missing values ignored. However, missing values at different positions have diverse influences on the distance measurement. This gap matters, which has been shown in prediction-related work [22], [23], but the general kNN considers all elements in the vectors equally.

\section{Methodology}

This section proposes a gap-sensitive windowed kNN for imputation to solve the issues mentioned above. Later, the data

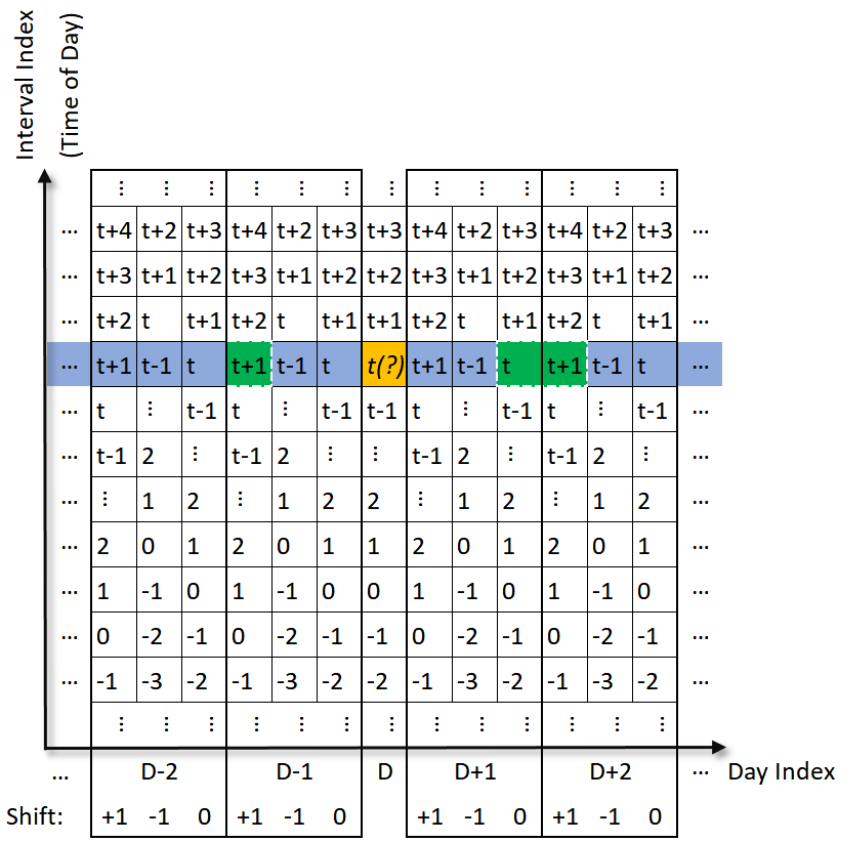

Fig. 2. Traffic prediction for time $t$ using GSW-kNN. The position of missing data is $(D, t)$. The shown example window is $v=1$, thus three shifts: $(-1,0,+1)$. Three dashed dark green squares are the selected nearest neighbours' data at $t$ or $t \pm 1$ of corresponding days $(k=3) .4$ searchable history days with three shifts lead to 12 potential neighbours. Besides, the instances indexes are extended into the neighbouring previous or later day when the lag $d$ is big.

and experimental setup are described.

\section{A. $G S W-k N N$}

As shown in Fig. 2, suppose the position of missing data is $(D, t)$ which is at the time $t$ on the day $D$. The search lag length is $d$ on both sides of $(D, t)$, so the state vector of the missing data is:

$$
\mathbf{r}_{D, t}=\left(r_{D, t-d}, \ldots, r_{D, t-1}, r_{D, t+1}, \ldots, r_{D, t+d}\right)
$$

where $\mathbf{r}$ means traffic flow volume rate. As an example, one searchable neighbour vector on the previous day $(D-1)$ without window is:

$$
\begin{aligned}
& \mathbf{r}_{D-1, t} \\
& =\left(r_{D-1, t-d}, \ldots, r_{D-1, t-1}, r_{D-1, t+1}, \ldots, r_{D-1, t+d}\right)
\end{aligned}
$$

where $\mathbf{r}_{D-1, t}$ is the matching point data. This step is similar to the general kNN neighbours except that the state vector of missing data and searchable history neighbours $\mathbf{r}$ can extend to neighbouring days when $d$ is big.

Here we add a window to the algorithm. A window $(v)$ is a limitation of shift that can be applied to all searchable history day and its matching point. For example, if $v=1$, the shift can be $-1,0,1$. If a shift 1 is applied to the neighbour vector $\mathbf{r}_{D-1, t}$ in (2), the neighbour vector becomes:

$$
\begin{aligned}
& \mathbf{r}_{D-1, t+1} \\
& =\left(r_{D-1, t-d+1}, \ldots, r_{D-1, t}, r_{D-1, t+2}, \ldots, r_{D-1, t+d+1}\right)
\end{aligned}
$$


Introducing window into $\mathrm{kNN}$ is a good way to make full use of data. Suppose the window size is $v$, then the shifts contained in the window are $-v,-v+1, \ldots,-1,0,1, \ldots, v-1, v$. Consequently, the non-zero shifts make the searchable dataset $v$ times bigger than the original dataset. This is important due to the fact that machine learning algorithms heavily rely on the big amount of available data. Especially when there is not too much data, such as only one single station/sensor's data are available.

The distance between neighbours is measured by Euclidean distance as it is frequently used in literature [24]. Other distance metrics such as Mahalanobis distance [25] or dynamic time warping [18], [26] are sometimes used but out of the scope of this work. Due to the fact that the search length $d$ is dynamic, using Euclidean distance is not suitable for $d$-dimension vectors. Also, to avoid the curse of dimension problem [27], we treat the time domain as one dimension instead of $d$-dimension by averaging the distance values from all search steps. The distance between the missing data vector (1) and one neighbour (2) is defined as follows:

$$
\dot{\mathbf{r}} \times \mathbf{w}=\left(\mathbf{r}_{D, t}-\mathbf{r}_{D, t-1}\right) \times \mathbf{w}
$$

where $\dot{\mathbf{r}}$ is the vector of absolute values of element-wise subtraction of $\mathbf{r}_{D, t}$ and $\mathbf{r}_{D, t-1}$. The $\mathbf{w}$ is the vector of weights for each search lag and it is gap-sensitive and is defined as follows.

Firstly, the initial scores are calculated:

$$
\begin{aligned}
\mathbf{w}^{*} & =\left(w_{1}^{*}, w_{2}^{*}, \ldots, w_{2 d}^{*}\right) \\
& =(1,2, \ldots, d-1, d, d-1, \ldots, 2,1)
\end{aligned}
$$

Those scores are given according to how far each element is differing from the missing data position in (1) or matching point position in (2). Later, the remaining values in $\mathbf{w}^{*}$ are normalized so that the sum of elements is 1 .

$$
\mathbf{w}=\frac{\mathbf{w}^{*}}{\sum_{i=1}^{2 d} w_{i}^{*}}
$$

It is worth mentioning that any values of missing positions in either vectors (1) or (2) are deleted from both vectors. If any element is deleted, both vectors should extend to include more values from further time points to keep the number of elements on both sides of the missing/matching point as $d$. This is because the missing data cannot be used to calculate distance.

\section{B. Data Specification}

The real world data is collected by the PeMS system traffic management centre in the Bay Area [28]. Each device sends one statistical record at five-minute intervals. Each record contains a timestamp and some statistical values such as the number of vehicles passed during the last five minutes, i.e. the flow rate. Part of the data from one monitoring device on the road is used, which is the public Dodgers dataset [29]. The time range of data is from April 2015 to October 2015.

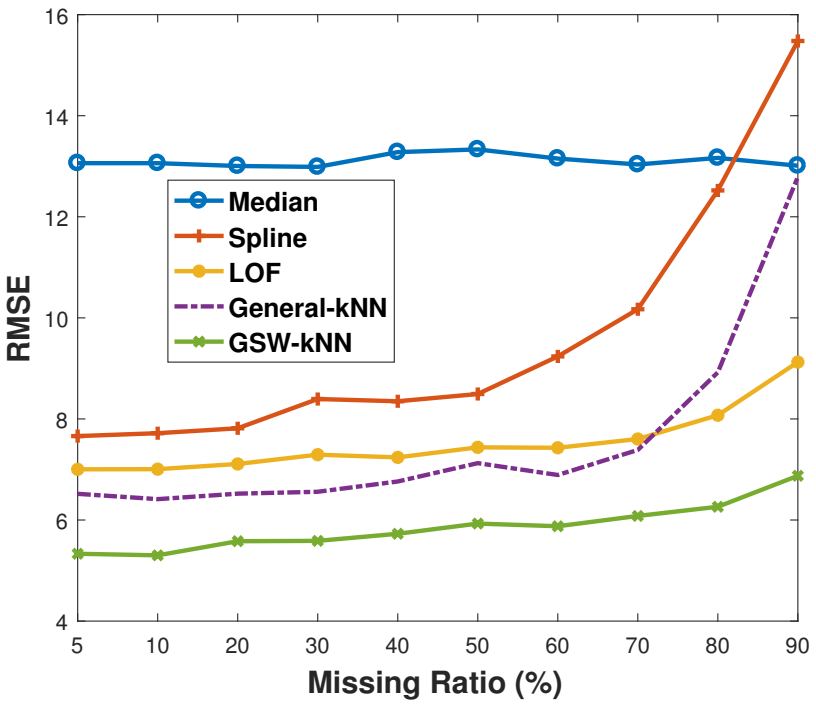

Fig. 3. RMSE of proposed GSW-kNN and four benchmarking methods. GSW-kNN is not only more accurate but also more stable when missing ratio is high.

The original dataset contains MCR and MR missing values which are $5 \%$. To test missing ratios higher than the original $5 \%$, MCR missing values are added.

\section{Experimental Setup}

The experiments are conducted using Python programming language version 2.7 [30] for GSW and with the main package impyute version 0.0.4 [31] for the general $\mathrm{kNN}$ method in Ubuntu 16.04 LTS. Other methods are conducted in $\mathrm{R}$ programming language version 3.3 .3 [32] and the package imputets version 2.5 [33] in Window 10. For each missing ratio, 5\% non-missing instances are selected randomly as test data and each of them is marked as missing during one experiment so that the influence the missing ratio is negligible. Best parameter configurations are used if needed.

The imputation accuracy is measured by root mean square error (RMSE), which is also known as root mean square deviation. RMSE is selected as it is frequently used in previous literature to measure imputation results so it is easier for readers to compare with previous work. Later, the imputation results are analyzed using cross comparison and eigenvalues.

\section{RESUlTS AND ANALYSIS}

This section describes benchmarking results among five methods, followed by more detailed comparison between two kNN based methods.

The RMSE comparison results of different methods are shown in Fig. 3. The missing ratio ranges from 5\% to $90 \%$. It is shown that RMSE values of GSW-kNN are the smallest compared to the other four methods. GSW-kNN performs $14 \%$ to $59 \%$ better than others and $34 \%$ better on average. Also, the RMSE values of GSW-kNN are very stable and the algorithm is robust. Except our proposed method, the general kNN method works better than the other three methods. 

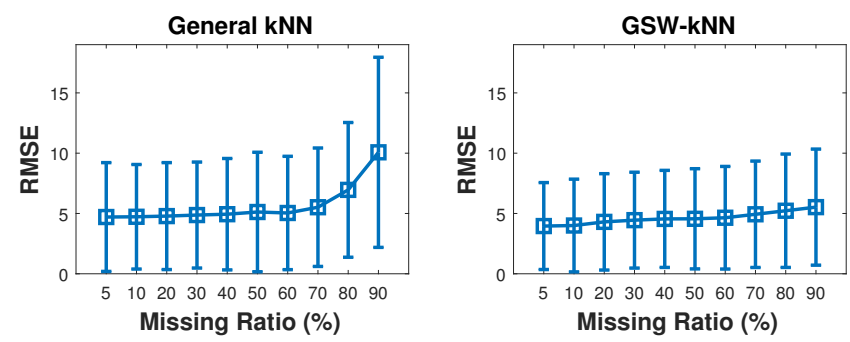

Fig. 4. RMSE and variation (standard deviation) of general kNN and GSW-kNN methods. GSW-kNN is more robust to high missing ratio.

Now we focus on the comparison of two kNN based methods. Fig. 4 shows the improvement of accuracy using our proposed method than the general kNN w.r.t. RMSE. GSW$\mathrm{kNN}$ is $18 \%$ to $46 \%$ better when being compared to general $\mathrm{kNN}$. The squares represent the mean values of RMSE for each missing ratio and the bars indicate standard deviation. As it is shown in Fig. 4, GSW-kNN is consistently better than general kNN. GSW-kNN has smaller RMSE on average and less variation (standard deviation), which means it works more stable from $5 \%$ to $90 \%$ missing ratio than the general $\mathrm{kNN}$ method.

The traffic data used in the experiments are distributed between 0 and 60. To understand more about how GSWkNN performs in different ranges of the traffic, several flow ranges are selected, say, 0 to 10,10 to 20,20 to 30 and 30 to 40.40 to 60 is not used due to very few data. In each range, 200 values are randomly selected. The improvement of RMSE from general kNN to the proposed GSW-kNN is shown in Fig. 5, indicating that in each of the four ranges, our proposed method performs better than general kNN with few exceptions. Especially, when the missing ratio is higher than $70 \%$, the accuracy improvement given by GSW-kNN increased dramatically in all the four ranges. Among the four ranges, the improvement in the range 0 to 10 is the highest. The reason is possibly that night traffic is more consistent across different days compared to day-time traffic which exhibits large variations during holidays. Thus, the windowed algorithm can make more use of existing night-time data [21].

Fig. 6 and Fig. 7 show the cross comparison plots of the ground truth and the imputed data from the general $\mathrm{kNN}$ and our proposed method. The $\mathrm{x}$-axis represents the ground truth values, and the y-axis represents imputed values. When the missing ratio is lower than $70 \%$, the shapes of point distributions from two methods are similar with each other, as well as among different missing ratios within the same method. This indicates that the two methods are both working in a stable way when the missing ratio is not too high. To describe the distribution of the cross comparison points in detail, the eigenvalues and eigenvectors of each covariance matrix is computed using singular value decomposition (SVD) method. According to the definition of the eigenvalues computed by SVD, the first eigenvalue depicts the data value range of the distribution, and the second eigenvalue represents the accuracy

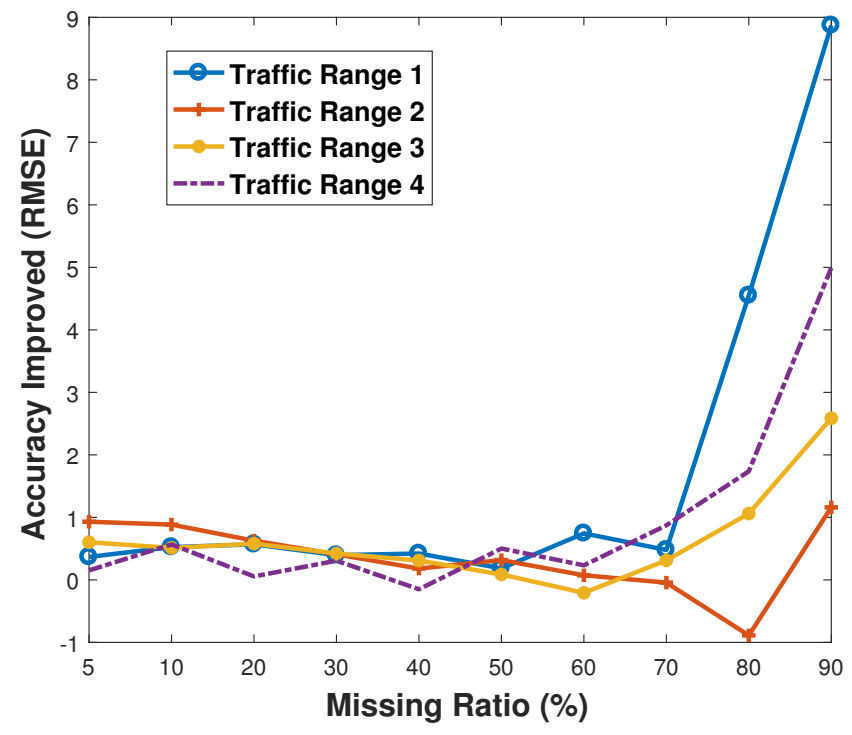

Fig. 5. The improvement of accuracy w.r.t. RMSE, when comparing GSW-kNN with general $\mathrm{kNN}$. GSW-kNN imputation is performing better especially in traffic flow range 1 (night time traffic) with high missing ratio.

of the data imputation. It means that the smaller the second eigenvalue is, the more accurate the imputation is. If the distribution of points is similar to a diagonal straight line, the imputed values are similar to the ground truth values.

Fig. 8 shows the second eigen values of the covariance matrix between ground truth value and the imputed value by the method of GSW-kNN and general kNN. Its results are consistent with our previous results discussion. Our proposed method, GSW-kNN works very stable and better than general $\mathrm{kNN}$.

Additionally, we also compared to PCA imputation from [34]. However, it cannot perform imputation for datasets with very high missing ratio, such as $80 \%$ and $90 \%$, so it is not included in the plots. The available results indicate that PCA is about $8 \%$ to $10 \%$ less accurate on average compared to GSW-kNN.

\section{CONCLuSion}

This paper proposes GSW-kNN for traffic time series imputation. The method takes gaps caused by missing values into consideration via using weights during distance measurement and uses windows to broaden the amount of neighbours. The experiment results from the public PeMS dataset are showing that GSW-kNN performs $14 \%$ to $59 \%$ better than benchmarking methods and $34 \%$ better on average. It is also more robust even for datasets with high missing ratios.

There are some issues that should be considered when using GSW-kNN. Firstly, more traditional time series based algorithms can be compared or used as part of ensemble methods as ensemble strategies are showing promising performance improvements compared to a single model for a variety of tasks [35], [36]. Secondly, our work does not distinguish data 

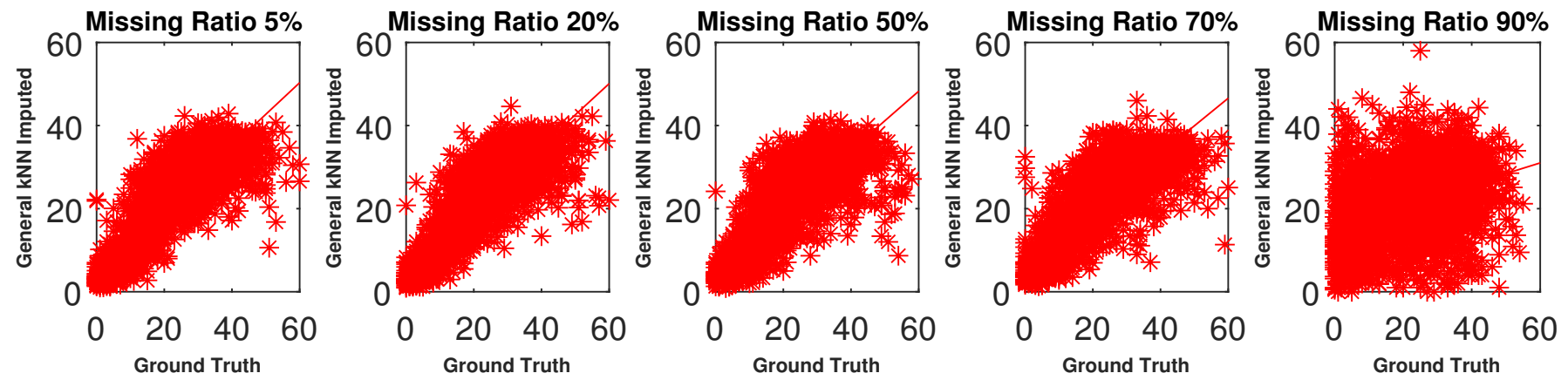

Fig. 6. The cross comparison between ground truth values and the imputed values by general kNN. Its performance drops suddenly when the missing ratio is higher than $70 \%$.
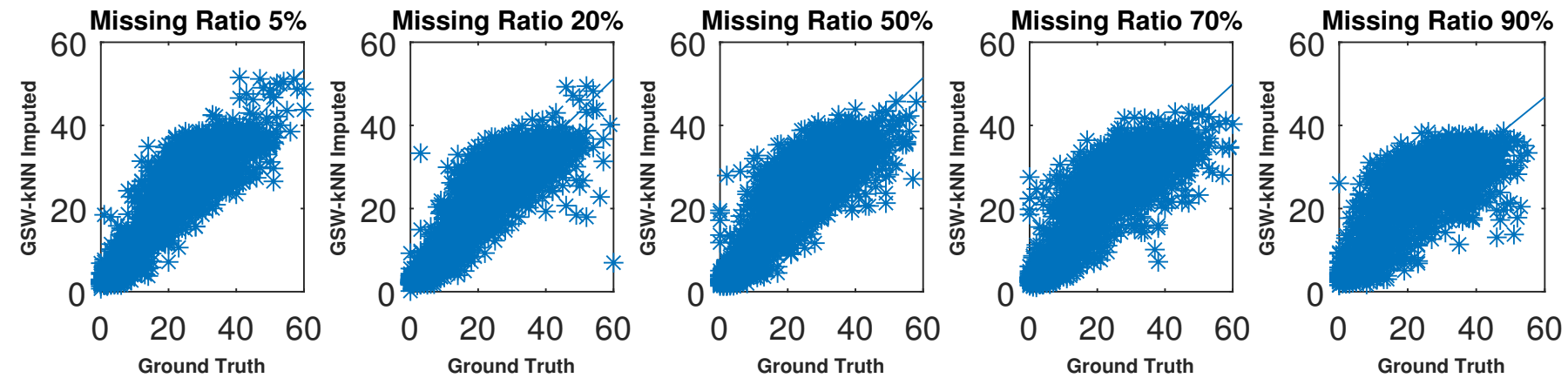

Fig. 7. The cross comparison between ground truth values and the imputed values by GSW-kNN. The distribution of points is more similar to a diagonal straight line than general kNN, especially when the missing ratio is higher than $70 \%$. Thus, GSW-kNN is more accurate.

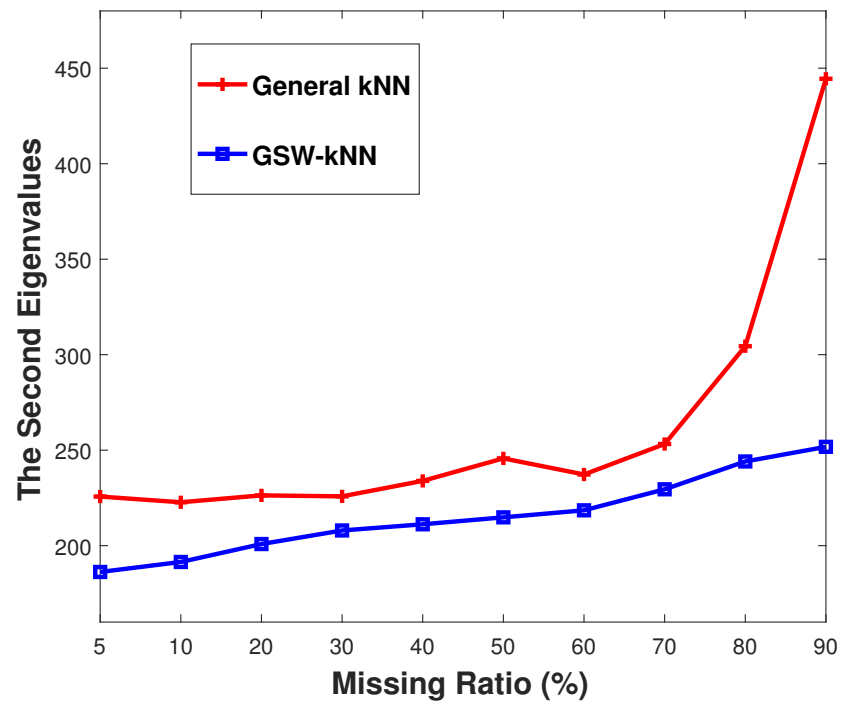

Fig. 8. The second eigenvalues of the cross comparison between ground truth values and the imputed values by the method of general $\mathrm{kNN}$ and GSW-kNN. GSW-kNN is giving smaller the second eigenvalues, hence the better imputation.

under normal situation with data under events. We plan to investigate those tasks in future work.

\section{ACKNOWLEDGMENT}

This work was supported by National Natural Science Foundation of China (NSFC), agreement 61364019.

\section{REFERENCES}

[1] J. Guo, W. Huang, and B. M. Williams, "Real time traffic flow outlier detection using short-term traffic conditional variance prediction," Transportation Research Part C: Emerging Technologies, vol. 50, pp. 160-172, Jan. 2015.

[2] M. Zhong, S. Sharma, and P. Lingras, "Genetically Designed Models for Accurate Imputation of Missing Traffic Counts," Transportation Research Record: Journal of the Transportation Research Board, vol. 1879, pp. 71-79, Jan. 2004.

[3] M. Moniruzzaman, H. Maoh, and W. Anderson, "Short-term prediction of border crossing time and traffic volume for commercial trucks: A case study for the Ambassador Bridge," Transportation Research Part C: Emerging Technologies, vol. 63, pp. 182-194, Feb. 2016.

[4] E. I. Vlahogianni, M. G. Karlaftis, and J. C. Golias, "Optimized and meta-optimized neural networks for short-term traffic flow prediction: A genetic approach," Transportation Research Part C-Emerging Technologies, vol. 13, no. 3, pp. 211-234, Jun. 2005.

[5] Y.-J. Byon and S. Liang, "Real-Time Transportation Mode Detection Using Smartphones and Artificial Neural Networks: Performance Comparisons Between Smartphones and Conventional Global Positioning System Sensors," Journal of Intelligent Transportation Systems, vol. 18, no. 3, pp. 264-272, Jul. 2014.

[6] M. Castro-Neto, Y.-S. Jeong, M.-K. Jeong, and L. D. Han, "Online-SVR for short-term traffic flow prediction under typical and atypical traffic conditions," Expert Systems with Applications, vol. 36, no. 3, Part 2, pp. 6164-6173, Apr. 2009.

[7] B. Sun, W. Cheng, P. Goswami, and G. Bai, "Short-Term Traffic Forecasting Using Self-Adjusting k-Nearest Neighbours," IET Intelligent Transport Systems, 2018.

[8] Y. Li, Z. Li, and L. Li, "Missing traffic data: Comparison of imputation methods," IET Intelligent Transport Systems, vol. 8, no. 1, pp. 51-57, Feb. 2014

[9] S. Tak, S. Woo, and H. Yeo, "Data-Driven Imputation Method for Traffic Data in Sectional Units of Road Links," IEEE Transactions on Intelligent Transportation Systems, vol. 17, no. 6, pp. 1762-1771, Jun. 2016. 
[10] L. Qu, L. Li, Y. Zhang, and J. Hu, "PPCA-Based Missing Data Imputation for Traffic Flow Volume: A Systematical Approach," IEEE Transactions on Intelligent Transportation Systems, vol. 10, no. 3, pp. 512-522, Sep. 2009.

[11] P. Loukopoulos, S. Sampath, P. Pilidis, G. Zolkiewski, I. Bennett, F. Duan, and D. Mba, "Dealing With Missing Data for Prognostic Purposes," in 2016 Prognostics and System Health Management Conference, M. J. Zuo, L. Xing, Z. Li, Z. Tian, and Q. Miao, Eds. New York: IEEE, 2016.

[12] A. N. Baraldi and C. K. Enders, "An introduction to modern missing data analyses," Journal of School Psychology, vol. 48, no. 1, pp. 5-37, Feb. 2010.

[13] P. Loukopoulos, G. Zolkiewski, I. Bennett, P. Pilidis, F. Duan, and D. Mba, "Dealing with missing data as it pertains of e-maintenance," Journal of Quality in Maintenance Engineering, no. just-accepted, pp. 00-00, 2017.

[14] Y. Li, Z. Li, L. Li, Y. Zhang, and M. Jin, "Comparison on PPCA, KPPCA and MPPCA Based Missing Data Imputing for Traffic Flow," in International Conference on Transportation Information and Safety (ICTIS). Wuhan, China: American Society of Civil Engineers, Jun. 2013.

[15] Y. Liu and V. Gopalakrishnan, "An Overview and Evaluation of Recent Machine Learning Imputation Methods Using Cardiac Imaging Data," Data, vol. 2, no. 1, p. 8, Jan. 2017.

[16] S. Chen and C. L. Yu, "Parameter estimation through semiparametric quantile regression imputation," Electronic Journal of Statistics, vol. 10, no. 2, pp. 3621-3647, 2016.

[17] G. Qin, J. Zhang, and Z. Zhu, "Simultaneous mean and covariance estimation of partially linear models for longitudinal data with missing responses and covariate measurement error," Computational Statistics \& Data Analysis, vol. 96, pp. 24-39, Apr. 2016.

[18] S. Oehmcke, O. Zielinski, and O. Kramer, "kNN Ensembles with Penalized DTW for Multivariate Time Series Imputation," in 2016 International Joint Conference on Neural Networks (IJCNN). New York: IEEE, 2016, pp. 2774-2781.

[19] Y. Rajabzadeh, A. H. Rezaie, and H. Amindavar, "Short-term traffic flow prediction using time-varying Vasicek model," Transportation Research Part C: Emerging Technologies, vol. 74, pp. 168-181, Jan. 2017.

[20] M. Bernas, B. Placzek, P. Porwik, and T. Pamula, "Segmentation of vehicle detector data for improved k-nearest neighbours-based traffic flow prediction," IET Intelligent Transport Systems, vol. 9, no. 3, pp. 264-274, Apr. 2015.

[21] B. Sun, W. Cheng, P. Goswami, and G. Bai, "Flow-Aware WPT kNearest Neighbours Regression for Short-Term Traffic Prediction," in IEEE Symposium on Computers and Communication (ISCC). Heraklion, Greece: IEEE, Jul. 2017, pp. 48-53.

[22] B. Sun, W. Cheng, P. Goswami, and G. Bai, "An Overview of Parameter and Data Strategies for k-Nearest Neighbours Based Short-Term Traffic Prediction," in International Conference on Intelligent Traffic and Transportation (ICITT). Zurich, Switzerland: IEEE, Sep. 2017.

[23] T. Kim, H. Kim, and D. J. Lovell, "Traffic flow forecasting: Overcoming memoryless property in nearest neighbor non-parametric regression," in 8th IEEE International Conference on Intelligent Transportation Systems (ITSC). Vienna, Austria: IEEE, Sep. 2005, pp. 965-969.

[24] B. Everitt, "Euclidean distance," in The Cambridge Dictionary of Statistics. Cambridge: Cambridge University Press, 2002, p. 134.

[25] B. Sun, W. Cheng, G. Bai, and P. Goswami, "Correcting and Complementing Freeway Traffic Accident Data Using Mahalanobis Distance Based Outlier Detection," Tehnicki Vjesnik-Technical Gazette, vol. 24, no. 5, Oct. 2017.

[26] S. Xu, B. Lu, M. Baldea, T. F. Edgar, W. Wojsznis, T. Blevins, and M. Nixon, "Data cleaning in the process industries," Reviews in Chemical Engineering, vol. 31, no. 5, pp. 453-490, Oct. 2015.

[27] V. Garcia, E. Debreuve, and M. Barlaud, "Fast k nearest neighbor search using GPU," in IEEE Computer Society Conference on Computer Vision and Pattern Recognition Workshops (CVPRW), Anchorage, AK, Jun. 2008, pp. 1-6.

[28] PeMS, "Dodgers Loop Sensor Data Set from Performance Measurement System," http://pems.dot.ca.gov/, Jul. 2017.

[29] A. Ihler, J. Hutchins, and P. Smyth, "Adaptive Event Detection with Time-varying Poisson Processes," in Proceedings of the 12th ACM SIGKDD International Conference on Knowledge Discovery and Data Mining, ser. KDD '06. New York, NY, USA: ACM, 2006, pp. 207-216.

[30] Python Software Foundation, "Python.Org," 2017.
[31] E. Law, "Impyute: Library of the different imputation algorithms; methods for dealing with ambiguity and handling missing data." 2017.

[32] R Core Team, R: A Language and Environment for Statistical Computing. Vienna, Austria: R Foundation for Statistical Computing, 2017.

[33] S. Moritz and T. Bartz-Beielstein, "imputeTS: Time Series Missing Value Imputation in R," $R$ Journal, vol. 9, no. 1, pp. 207-218, Jun. 2017.

[34] J. Josse and F. Husson, "Handling missing values in exploratory multivariate data analysis methods," Journal de la Societe Francaise de Statistique, vol. 153, no. 2, pp. 79-99, Dec. 2012.

[35] L. Ma, B. Sun, and L. Ziyi, "Bagging Likelihood-Based Belief Decision Trees," in 20th International Conference on Information Fusion (FUSION), Xi-An, China, Jul. 2017, pp. 1-6.

[36] T. A. Moahmed, N. E. Gayar, and A. F. Atiya, "Forward and Backward Forecasting Ensembles for the Estimation of Time Series Missing Data," in IAPR Workshop on Artificial Neural Networks in Pattern Recognition, ser. Lecture Notes in Computer Science. Springer, Cham, Oct. 2014, pp. 93-104. 